\title{
Self-error-rejecting photonic qubit transmission in polarization-spatial modes with linear optical elements*
}

\author{
Yu-Xiao Jiang ${ }^{1}$, Peng-Liang Guo ${ }^{1}$, Cheng-Yan Gao ${ }^{1}$, Hai-Bo \\ Wang $^{1}$, Faris Alzahrani ${ }^{2}$, Aatef Hobiny ${ }^{2}$, Fu-Guo Deng ${ }^{1,2, \dagger}$ \\ ${ }^{1}$ Department of Physics, Applied Optics Beijing Area Major Laboratory, \\ Beijing Normal University, Beijing 100875, China \\ ${ }^{2}$ NAAM-Research Group, Department of Mathematics, Faculty of Science, \\ King Abdulaziz University, P.O. Box 80203, Jeddah 21589, Saudi Arabia
}

(Dated: July 15, 2021)

\begin{abstract}
We present an original self-error-rejecting photonic qubit transmission scheme for both the polarization and spatial states of photon systems transmitted over collective noise channels. In our scheme, we use simple linear-optical elements, including half-wave plates, 50:50 beam splitters, and polarization beam splitters, to convert spatial-polarization modes into different time bins. By using postselection in different time bins, the success probability of obtaining the uncorrupted states approaches $1 / 4$ for single-photon transmission, which is not influenced by the coefficients of noisy channels. Our self-error-rejecting transmission scheme can be generalized to hyperentangled $\mathrm{N}$-photon systems and is useful in practical high-capacity quantum communications with photon systems in two degrees of freedom.
\end{abstract}

Keywords: Photon transmission · Error correction $\cdot$ Collective-noise channel $\cdot$ Spatial-polarization modes · Quantum communication

PACS numbers: 03.67.Pp, 03.67.Dd, 03.67.Hk

\section{INTRODUCTION}

A photon can serve as an ideal qubit because of its maneuverability; thus, it is regarded as an essential quantum system for realizing quantum communication, including quantum teleportation [1, 2], quantum dense coding 3, [4], quantum key distribution [5 [8], quantum secret sharing [9], and quantum secure direct communication [10 16]. However, during transmission over an optical-fiber or free-space channel, photons are inevitably influenced by the thermal fluctuations, vibrations, and imperfections of the fiber, i.e., noise in the quantum channel [17-19], leading to phase shifts in spatial modes and changes in polarization states. Error-rejecting qubit transmission is an effective method to resist the influence of collective noise [19]. In 2005, Kalamidas [17] proposed two linear-optical singlephoton schemes to reject and correct arbitrary qubit errors without additional qubits. In the same year, Yamamoto et al. [18] developed a single-photon error-rejecting scheme with an ancillary qubit in fixed polarization. In this scheme, an ancillary particle and a photon in an arbitrary quantum state pass through the same apparatus and are affected by the noise channel identically. Detection of the outports of two photons could exclude errors, thereby probabilistically giving the original quantum state, the fidelity of which is 1 . The proposed scheme presented a low success probability, which is relevant to the parameters of noise. Application of photon detectors and a two-qubit entanglement gate yielded a success probability of only 1/8. In 2007, Li et al. [19] presented a very useful scheme for passively error-rejecting qubit transmission using linear optics and postselection in different time bins without ancillary qubits. In their scheme, qubits were encoded in time bins, and uncorrupted photons arrived at definite time slots; this scheme promised a $50 \%$ success probability in the case of linear-optical elements only. If time delayers were applied, the success probability could approach 100\%, regardless of the coefficients of noise.

Hyperentanglement, which is defined as the simultaneous entanglement in two or more degrees of freedom (DOF) of a photon system 20 26], has attracted great attention recently as it can improve the capacity of quantum communication 27 32 and speed up the quantum computation [33 36]. For example, it can be used to complete the task of teleporting an unknown quantum state in more than one DOF and double the channel capacity of quantum communication via the complete hyperentangled Bell-state analysis 27-32]. Hyperentanglement can also be used to beat the channel capacity limit for linear photonic superdense coding [37], achieve the deterministic entanglement purification [3842], and construct high-efficiency quantum repeaters [43]. In practical applications of hyperentanglement in highcapacity quantum communication, hyperentangled photon systems suffer from channel noise, as well as photonic

\footnotetext{
* published in Sci. China-Phys. Mech. Astron. 60, 120312 (2017)
}

$\dagger$ Corresponding author: fgdeng@bnu.edu.cn 
quantum states with one DOF. Two approaches can depress the influence of noise on hyperentangled quantum systems. The first approach is the passive method, including hyperentanglement purification [44 48] and hyperentanglement concentration [49 58], through which the parties in long-distance quantum communication can distill some highfidelity hyperentangled photon systems from nonlocal less-hyperentanglement systems polluted by channel noise. The second approach is the active method, including error-rejecting qubit transmission and error-correcting code. This method uses special DOFs of photon systems or decoherence-free subspace to protect photonic systems when they are transmitted over a collective noise channel. These two approaches are necessary to overcome the influence of channel noise in practical long-distance quantum communication. To date, no works on error-rejecting qubit transmissions for hyperentangled photon systems with linear-optical elements have been published.

In this paper, we first present an original scheme for self-error-rejecting single-qubit transmission for photon systems in both the spatial and polarization modes using linear-optical elements only. The system consists of an unbalanced polarization interferometer based on a polarizing beam splitter, a 50:50 beam splitter, and a half-wave plate. We also apply several additional BSs to overcome the phase shift in the spatial-mode DOF. For a single-photon transmission, the success probability of receiving uncorrupted photons is $25 \%$. We apply this method to the transmission of two hyperentangled photons and entangled n-photons and demonstrate its applicability to future high-capacity quantum communication.

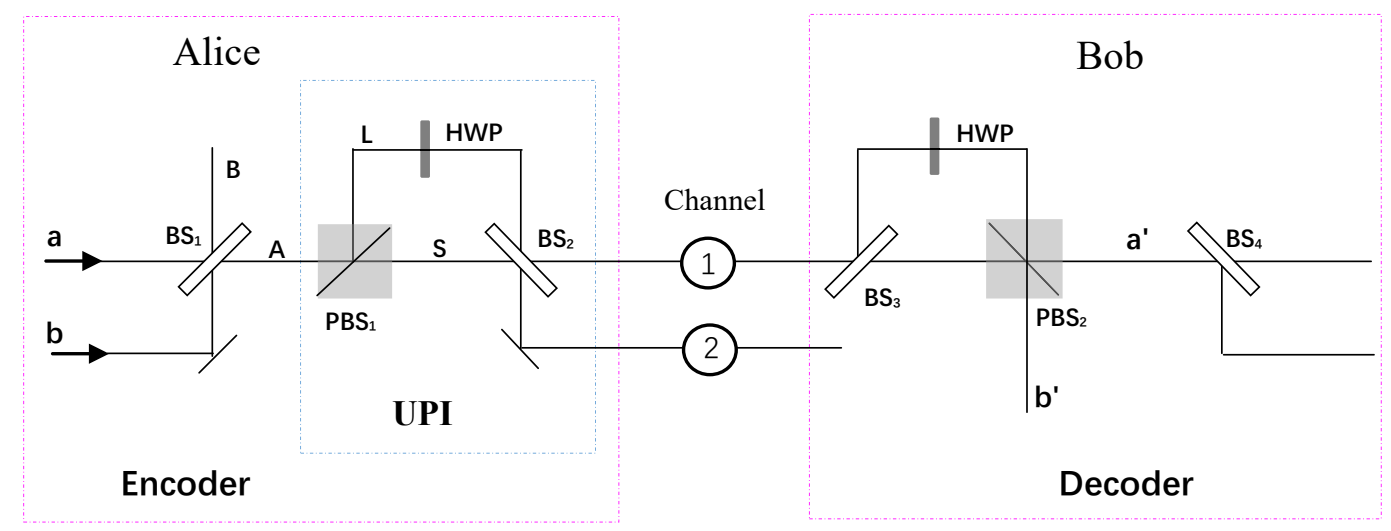

FIG. 1: Schematic diagram of the error-correcting distribution of a single-photon in polarization-spatial modes. $a$ and $b$ represent the two spatial modes of the photon. $A$ and $B$ represent two different paths for the photon. PBS $i(i=1,2)$ represents a polarizing beam splitter, which transmits photons with a horizontal polarization and reflects photons with a vertical polarization. $\mathrm{BS}_{j}(j=1,2,3,4)$ is a 50:50 beam splitter. HWP denotes a half-wave plate which is used to complete the transformations between the polarizations $|V\rangle$ and $|H\rangle$, i.e., $|V\rangle \rightleftarrows|H\rangle$. UPI represents an unbalanced polarizing interferometer.

\section{ERROR-REJECTING SINGLE-PHOTON TRANSMISSION IN POLARIZATION-SPATIAL MODES}

We suppose that the transmitted photon $P$ from Alice is in an arbitrary polarization-spatial state

$$
|\Psi\rangle_{P}=\left(\alpha^{\prime}|a\rangle+\beta^{\prime}|b\rangle\right) \otimes(\alpha|H\rangle+\beta|V\rangle) .
$$

Here $|\alpha|^{2}+|\beta|^{2}=\left|\alpha^{\prime}\right|^{2}+\left|\beta^{\prime}\right|^{2}=1 .|H\rangle$ and $|V\rangle$ represent the horizontal and vertical polarizations of photons, respectively. $|a\rangle$ and $|b\rangle$ denote the different spatial modes of the photon $P$. The principle of our scheme for the errorrejecting single-photon transmission in polarization-spatial modes is shown in Fig.1. The photon $P$ in the spatial mode $|b\rangle$ apparently passes through a long path, causing a time delay in the order of a few nanoseconds. We represent the time difference between the two spatial modes $|a\rangle$ and $|b\rangle$ by $\delta t . \mathrm{BS}_{1}$ redistributes the paths a and $\mathrm{b}$ to two new paths $\mathrm{A}$ and $\mathrm{B}$, and the state of the photon $P$ after it is divided into $\mathrm{A}$ and $\mathrm{B}$ can be described as follows:

$$
\begin{aligned}
|\Psi\rangle_{P} & =\left(\alpha^{\prime}|a\rangle+\beta^{\prime}|b\rangle\right) \otimes(\alpha|H\rangle+\beta|V\rangle) \\
& \stackrel{B S_{1}}{\longrightarrow} \frac{1}{\sqrt{2}}\left(\alpha^{\prime}|a\rangle_{0}+i \beta^{\prime}|b\rangle_{\delta t}\right)(\alpha|H\rangle+\beta|V\rangle)_{A}+\frac{1}{\sqrt{2}}\left(\beta^{\prime}|b\rangle_{\delta t}+i \alpha^{\prime}|a\rangle_{0}\right)(\alpha|H\rangle+\beta|V\rangle)_{B} \\
& \equiv|\psi\rangle_{A}+|\psi\rangle_{B} .
\end{aligned}
$$


Here

$$
\begin{aligned}
|\psi\rangle_{A} & =\frac{1}{\sqrt{2}}\left(\alpha^{\prime}|a\rangle_{0}+i \beta^{\prime}|b\rangle_{\delta t}\right)(\alpha|H\rangle+\beta|V\rangle)_{A}, \\
|\psi\rangle_{B} & =\frac{1}{\sqrt{2}}\left(\beta^{\prime}|b\rangle_{\delta t}+i \alpha^{\prime}|a\rangle_{0}\right)(\alpha|H\rangle+\beta|V\rangle)_{B} .
\end{aligned}
$$

In either path $\mathrm{A}$ or $\mathrm{B}$, states $|a\rangle$ and $|b\rangle$ are distinguished by a time delay. In addition, the quantum states in these two paths can be transformed into each other by a unitary operation; thus, we only consider the outcome from path $\mathrm{A}$ in the following discussion. The outcome from path B is similar to that from path $\mathrm{A}$.

The photon in path A passes through an UPI. $\mathrm{PBS}_{1}$ in the encoding system transmits the horizontal polarization mode $|H\rangle$ and reflects the vertical polarization mode $|V\rangle$, thus allowing states $|H\rangle$ and $|V\rangle$ to propagate through the short path $S$ and the long path $L$, respectively. This length difference also results in a time delay in the order of a few nanoseconds. The HWP transforms $|V\rangle$ to $|H\rangle$. The transformation of the state for the photon $\mathrm{P}$ in path A before it enters the noisy channel could be described as follows:

$$
\begin{aligned}
|\psi\rangle_{A} & =\frac{1}{\sqrt{2}}\left(\alpha^{\prime}|a\rangle_{0}+i \beta^{\prime}|b\rangle_{\delta t}\right)(\alpha|H\rangle+\beta|V\rangle) \\
\stackrel{P B S_{1}}{\longrightarrow} & \frac{1}{\sqrt{2}}\left(\alpha^{\prime}|a\rangle_{0}+i \beta^{\prime}|b\rangle_{\delta t}\right)\left(\alpha|H\rangle_{S}+\beta|H\rangle_{L}\right) \\
\stackrel{B S_{2}}{\longrightarrow} & \frac{1}{2}\left(\alpha^{\prime}|a\rangle_{0}+i \beta^{\prime}|b\rangle_{\delta t}\right)\left[\left(\alpha|H\rangle_{S}+i \beta|H\rangle_{L}\right)_{1}+\left(i \alpha|H\rangle_{S}+\beta|H\rangle_{L}\right)_{2}\right] \\
\equiv & |\psi\rangle_{1}+|\psi\rangle_{2} .
\end{aligned}
$$

Here

$$
\begin{aligned}
|\psi\rangle_{1} & =\frac{1}{2}\left(\alpha^{\prime}|a\rangle_{0}+i \beta^{\prime}|b\rangle_{\delta t}\right)\left(\alpha|H\rangle_{S}+i \beta|H\rangle_{L}\right)_{1}, \\
|\psi\rangle_{2} & =\frac{1}{2}\left(\alpha^{\prime}|a\rangle_{0}+i \beta^{\prime}|b\rangle_{\delta t}\right)\left(i \alpha|H\rangle_{S}+\beta|H\rangle_{L}\right)_{2} .
\end{aligned}
$$

Two output ports from $\mathrm{BS}_{2}$ are denoted by the subscripts 1 and 2, as shown in Fig. 1. Similar to the previous analysis for paths $\mathrm{A}$ and $\mathrm{B}$, we only consider channel 1 for simplicity. Because both periods of time delay mentioned above are much less than the phase shift, we can assume that the infection caused by noise is the same for different parts. The change of states could be described by a unitary operation and an overall phase shift

$$
\begin{aligned}
& |\psi\rangle \stackrel{\text { channel }}{\longrightarrow} e^{i \theta}|\psi\rangle, \\
& |H\rangle \stackrel{\text { channel }}{\longrightarrow} \gamma|H\rangle+\eta|V\rangle,
\end{aligned}
$$

where $|\gamma|^{2}+|\eta|^{2}=1$. Then through channel 1 , the evolution of the state could be written as

$$
\begin{aligned}
|\psi\rangle_{1} \quad=\quad & \frac{1}{2}\left(\alpha^{\prime}|a\rangle_{0}+i \beta^{\prime}|b\rangle_{\delta t}\right)\left(\alpha|H\rangle_{S}+i \beta|H\rangle_{L}\right) \\
\stackrel{\text { channel }}{\longrightarrow} & \frac{1}{2} e^{i \theta}\left(\alpha^{\prime}|a\rangle_{0}+i \beta^{\prime}|b\rangle_{\delta t}\right)\left(\gamma \alpha|H\rangle_{S}+\eta \alpha|V\rangle_{S}+i \gamma \beta|H\rangle_{L}+i \eta \beta|V\rangle_{L}\right) .
\end{aligned}
$$

The decoding system, which is symmetric to the encoding system, can transform the photon state from channel 1 , which is noisy, as follows:

$$
\begin{aligned}
\left|\psi^{\prime}\right\rangle_{1} \stackrel{B S_{3}}{\longrightarrow H W P^{\prime}} & \frac{1}{2 \sqrt{2}} e^{i \theta}\left(\alpha^{\prime}|a\rangle_{0}+i \beta^{\prime}|b\rangle_{\delta t}\right)\left(\alpha \gamma|H\rangle_{S S}+\alpha \eta|V\rangle_{S S}+i \beta \gamma|H\rangle_{L S}+i \beta \eta|V\rangle_{L S}\right) \\
& +\frac{1}{2 \sqrt{2}} e^{i \theta}\left(\alpha^{\prime}|a\rangle_{0}+i \beta^{\prime}|b\rangle_{\delta t}\right)\left(\alpha \gamma i|V\rangle_{S L}+\alpha \eta i|H\rangle_{S L}-\beta \gamma|V\rangle_{L L}-\beta \eta|H\rangle_{L L}\right) \\
\stackrel{P B S_{2}}{\longrightarrow} & \frac{\gamma}{2 \sqrt{2}} e^{i \theta}\left(\alpha^{\prime}|a\rangle_{0}+i \beta^{\prime}|b\rangle_{\delta t}\right)\left[\alpha|H\rangle_{S S}-\beta|V\rangle_{L L}+i \underline{\left(\alpha|V\rangle_{S L}+\beta|H\rangle_{L S}\right)}\right]_{a^{\prime}} \\
& +\frac{\eta}{2 \sqrt{2}} e^{i \theta}\left(\alpha^{\prime}|a\rangle_{0}+i \beta^{\prime}|b\rangle_{\delta t}\right)\left[\alpha|V\rangle_{S S}-\beta|H\rangle_{L L}+i \underline{\left(\alpha|H\rangle_{S L}+\beta|V\rangle_{L S}\right)}\right]_{b^{\prime}} \\
\stackrel{B S_{4}}{\longrightarrow} & \frac{\gamma}{4} e^{i \theta}\left[\alpha^{\prime}|a\rangle_{0}-\beta^{\prime}|b\rangle_{2 \delta t}+i \underline{\left(\alpha^{\prime}|a\rangle_{\delta t}+\beta^{\prime}|b\rangle_{\delta t}\right)}\right]\left[\alpha|H\rangle_{S S}-\beta|V\rangle_{L L}+i \underline{\left(\alpha|V\rangle_{S L}+\beta|H\rangle_{L S}\right)}\right]_{a^{\prime}} \\
& +\frac{\eta}{4} e^{i \theta}\left[\alpha^{\prime}|a\rangle_{0}-\beta^{\prime}|b\rangle_{2 \delta t}+\underline{i\left(\alpha^{\prime}|a\rangle_{\delta t}+\beta^{\prime}|b\rangle_{\delta t}\right)}\right]\left[\alpha|V\rangle_{S S}-\beta|H\rangle_{L L}+i \underline{\left(\alpha|H\rangle_{S L}+\beta|V\rangle_{L S}\right)}\right]_{b^{\prime}}
\end{aligned}
$$


The subscripts $a^{\prime}$ and $b^{\prime}$ label two outputs from the UPI system, and the states in two paths differ by a unitary transformation.

Through the last BS, the two spatial modes are separated once more, as displayed in Fig. 1. As long as the time delay caused by $\mathrm{BS}_{4}$ is equal to the initial time delay $\delta t$, states in the spatial mode will arrive at three definite times. Among these times, we only consider the states in the middle time, which carries uncorrupted information about the original quantum state. The same method could be used in the analysis of polarization modes, where we will focus on states in the $L S$ and $S L$ modes. Moreover, the time delay caused by the UPI system is at least two times larger than $\delta t$ in the case of the interference of two DOFs. The uncorrupted states are underlined in Equation (8). Especially, for port $a^{\prime}$, a bit-flip operation on the polarization DOF is necessary to achieve the initial polarization state.

The success probability of obtaining the uncorrupted state for each of the ports $a^{\prime}$ and $b^{\prime}$ is $\frac{1}{16}$. Notice that two main paths $\mathrm{A}$ and $\mathrm{B}$ are separately distributed to two channels. The total success probability is $\frac{1}{4}$.

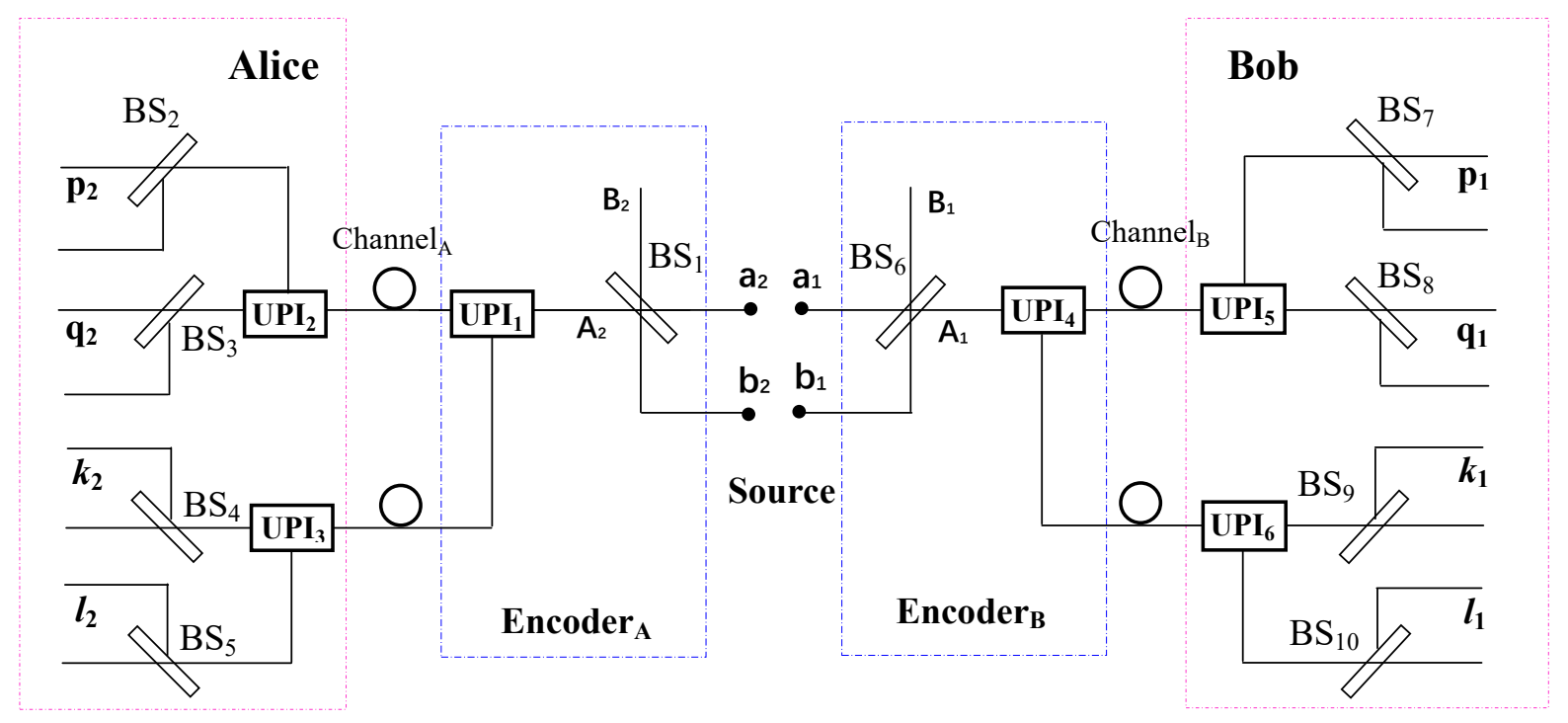

FIG. 2: Schematic diagram of the error-correcting distribution of a pair of hyperentangled photons with spatial-polarization DOFs. $a_{j}$ and $b_{j}$ represent the two spatial modes of the photon $j(j=1,2) . A_{j}$ and $B_{j}$ are two different paths for the photon $j$. UPI is an unbalanced polarizing interferometer, as shown in Fig. 1. The circles represent the channels over which the photons are separately transmitted to the two parties Alice and Bob. The hyperentangled photon pair 12 in the state $|\psi\rangle=\left(\alpha^{\prime}\left|a_{1} a_{2}\right\rangle+\beta^{\prime}\left|b_{1} b_{2}\right\rangle\right)_{12}(\alpha|H H\rangle+\beta|V V\rangle)_{12}$ is produced from the quantum source in the middle node and the two photons 1 and 2 are transmitted to the two parties Bob and Alice, respectively. Before the photons are transmitted over the optical-fiber channels, encoders are used to code their quantum states. After the parties Alice and Bob receive the photons, they decode the quantum state with decoders.

\section{ERROR-REJECTING TRANSMISSION OF TWO ENTANGLED PHOTONS IN SPATIAL-POLARIZATION HYPERENTANGLED MODES}

Now, let us consider the error-rejecting transmission of a pair of hyperentangled photons. The schematic diagram of our scheme for the error-correcting distribution of a pair of hyperentangled photons with spatial-polarization DOFs is shown in Fig. 2, Two entangled photons marked 1 and 2 are distributed to two identical encoding and decoding systems and arrive at four possible ports $p, q, k$, and $l$, respectively. Suppose that the initial state is described by $|\psi\rangle=\left(\alpha^{\prime}\left|a_{1} a_{2}\right\rangle+\beta^{\prime}\left|b_{1} b_{2}\right\rangle\right)_{12}(\alpha|H H\rangle+\beta|V V\rangle)_{12}$.

In the following discussion, we will analyze the transformation of the states $|a\rangle,|b\rangle,|H\rangle$, and $|V\rangle$, separately. The effect of $\mathrm{BS}_{1}$ and $\mathrm{BS}_{6}$ in Fig. 2 can be regarded as adding a Hadamard operation to photons in the spatial mode. 
The change in the states $|a\rangle\left(\left|a_{1}\right\rangle\right.$ and $\left.\left|a_{2}\right\rangle\right)$ and $|b\rangle\left(\left|b_{1}\right\rangle\right.$ and $\left.\left|b_{2}\right\rangle\right)$ could be written as

$$
\begin{aligned}
|a\rangle \stackrel{B S}{\longrightarrow} \frac{1}{\sqrt{2}}\left(|a\rangle_{A}+i|a\rangle_{B}\right) \stackrel{\text { channel }}{\longrightarrow} \frac{1}{\sqrt{2}}\left(e^{i \theta_{A}}|a\rangle_{A}+i e^{i \theta_{B}}|a\rangle_{B}\right) \\
\stackrel{B S}{\longrightarrow} \frac{1}{2}\left(e^{i \theta_{A}}|a\rangle_{A}+i e^{i \theta_{B}}|a\rangle_{B}+\underline{i e^{i \theta_{A}}|a\rangle_{\delta t A}-e^{i \theta_{B}}|a\rangle_{\delta t B}}\right), \\
|b\rangle \stackrel{B S}{\longrightarrow} \frac{1}{\sqrt{2}}\left(i|b\rangle_{\delta t A}+|b\rangle_{\delta t B}\right) \stackrel{\text { channel }}{\longrightarrow} \frac{1}{\sqrt{2}}\left(i e^{i \theta_{A}}|b\rangle_{\delta t A}+e^{i \theta_{B}}|b\rangle_{\delta t B}\right) \\
\stackrel{B S}{\longrightarrow} \frac{1}{2}\left(i e^{i \theta_{B}}|b\rangle_{2 \delta t B}-e^{i \theta_{A}}|b\rangle_{2 \delta t A}+\underline{i e^{i \theta_{A}}|b\rangle_{\delta t A}+e^{i \theta_{B}}|b\rangle_{\delta t B}} .\right.
\end{aligned}
$$

Terms with the time delay $\delta t$ are underlined because they are the states from which we will extract information about the initial quantum state. Applying Equation (9), we can obtain an expression of the ultimate spatial states of the hyperentangled photons:

$$
\begin{aligned}
& \alpha^{\prime}\left|a_{1} a_{2}\right\rangle+\beta^{\prime}\left|b_{1} b_{2}\right\rangle \\
\rightarrow & \frac{\alpha^{\prime}}{4}\left(i e^{i \theta_{A_{1}}}\left|a_{1}\right\rangle_{\delta t A^{1}}-e^{i \theta_{B_{1}}}\left|a_{2}\right\rangle_{\delta t B^{1}}\right)\left(i e^{i \theta_{A_{2}}}\left|a_{1}\right\rangle_{\delta t A^{2}}-e^{i \theta_{B_{2}}}\left|a_{2}\right\rangle_{\delta t B^{2}}\right) \\
& +\frac{\beta^{\prime}}{4}\left(i e^{i \theta_{A_{1}}}\left|b_{1}\right\rangle_{\delta t A^{1}}+e^{i \theta_{B_{1}}}\left|b_{2}\right\rangle_{\delta t B^{1}}\right)\left(i e^{i \theta_{A_{2}}}\left|b_{1}\right\rangle_{\delta t A^{2}}+e^{i \theta_{B_{2}}}\left|b_{2}\right\rangle_{\delta t B^{2}}\right) \\
= & \frac{i}{4} e^{i\left(\theta_{A_{1}}+\theta_{B_{2}}\right)}\left(-\alpha^{\prime}\left|a_{1}\right\rangle_{\delta t A^{1}}\left|a_{2}\right\rangle_{\delta t B^{2}}+\beta^{\prime}\left|b_{1}\right\rangle_{\delta t A^{1}}\left|b_{2}\right\rangle_{\delta t B^{2}}\right) \\
& +\frac{1}{4} e^{i\left(\theta_{A_{1}}+\theta_{A_{2}}\right)}\left(-\alpha^{\prime}\left|a_{1}\right\rangle_{\delta t A^{1}}\left|a_{2}\right\rangle_{\delta t A^{2}}-\beta^{\prime}\left|b_{1}\right\rangle_{\delta t A^{1}}\left|b_{2}\right\rangle_{\delta t A^{2}}\right) \\
& +\frac{i}{4} e^{i\left(\theta_{B_{1}}+\theta_{A_{2}}\right)}\left(-\alpha^{\prime}\left|a_{1}\right\rangle_{\delta t B^{1}}\left|a_{2}\right\rangle_{\delta t A^{2}}+\beta^{\prime}\left|b_{1}\right\rangle_{\delta t B^{1}}\left|b_{2}\right\rangle_{\delta t A^{2}}\right) \\
& +\frac{1}{4} e^{i\left(\theta_{B_{1}}+\theta_{B_{2}}\right)}\left(\alpha^{\prime}\left|a_{1}\right\rangle_{\delta t B^{1}}\left|a_{2}\right\rangle_{\delta t B^{2}}+\beta^{\prime}\left|b_{1}\right\rangle_{\delta t B^{1}}\left|b_{2}\right\rangle_{\delta t B^{2}}\right)
\end{aligned}
$$

For simplicity, we neglect the states that arrive too late $(2 \delta t)$ or too early. The success probability of receiving the uncorrupted states in spatial modes is $\frac{1}{4}$ in total, which is clearly displayed in Equation (10). For each port, a definite unitary operation must be applied to rebuild the original state.

The same approach could be applied to the analysis of polarization states, which is performed as follows:

$$
\begin{aligned}
&|H\rangle \quad \stackrel{U P I}{\longrightarrow} \frac{1}{\sqrt{2}}\left(|H\rangle_{S_{1}}+i|H\rangle_{S_{2}}\right) \\
& \stackrel{\text { channel }}{\longrightarrow} \frac{1}{\sqrt{2}}\left(\gamma|H\rangle_{S}+\eta|V\rangle_{S}+i \gamma^{\prime}|H\rangle_{S}+i \eta^{\prime}|V\rangle_{S}\right) \\
& \quad \stackrel{U P I}{\longrightarrow} \frac{1}{2}\left[\gamma\left(|H\rangle_{S S}+i|V\rangle_{S L}\right)_{p}+\eta\left(|V\rangle_{S S}+|H\rangle_{S L}\right)_{q}+i \gamma^{\prime}\left(|H\rangle_{S S}+|V\rangle_{S L}\right)_{k}+i \eta^{\prime}\left(|V\rangle_{S S}+|H\rangle_{S L}\right)_{l}\right] \\
& \quad \stackrel{\text { channel }}{\longrightarrow} \frac{1}{\sqrt{2}}\left(\gamma^{\prime}|H\rangle_{L}+\eta^{\prime}|V\rangle_{L}+i \gamma|H\rangle_{L}+i \eta|V\rangle_{L}\right) \\
& \quad \stackrel{\frac{U P I}{\longrightarrow}}{\longrightarrow} \frac{1}{2}\left[\gamma\left(i|H\rangle_{L S}-|V\rangle_{L L}\right)_{p}+\eta\left(i|V\rangle_{L S}-|H\rangle_{L L}\right)_{q}+\gamma^{\prime}\left(|H\rangle_{L S}+i|V\rangle_{L L}\right)_{k}+\eta^{\prime}\left(|V\rangle_{L S}+i|H\rangle_{L L}\right)_{l}\right]
\end{aligned}
$$

Notice that $\eta, \gamma$ and $\eta^{\prime}, \gamma^{\prime}$ describe the effect of the collective noise from different channels satisfying $|\eta|^{2}+|\gamma|^{2}=$ 
$\left|\eta^{\prime}\right|^{2}+\left|\gamma^{\prime}\right|^{2}=1$. Again we only focus on those terms with the subscripts $S L$, and obtain the uncorrupted states

$$
\begin{aligned}
& \alpha|H H\rangle+\beta|V V\rangle \\
\rightarrow & \frac{\alpha}{4}\left(i \gamma_{1}|V\rangle_{p_{1}}+\eta_{1}|H\rangle_{q_{1}}+i \gamma_{1}^{\prime}|V\rangle_{k_{1}}+i \eta_{1}^{\prime}|H\rangle_{l_{1}}\right)\left(i \gamma_{2}|V\rangle_{p_{2}}+\eta_{2}|H\rangle_{q_{2}}+i \gamma_{2}^{\prime}|V\rangle_{k_{2}}+i \eta_{2}^{\prime}|H\rangle_{l_{2}}\right) \\
& +\frac{\beta}{4}\left(i \gamma_{1}|H\rangle_{p_{1}}+i \eta_{1}|V\rangle_{q_{1}}+\gamma_{1}^{\prime}|H\rangle_{k_{1}}+\eta_{1}^{\prime}|V\rangle_{l_{1}}\right)\left(i \gamma_{2}|H\rangle_{p_{2}}+i \eta_{2}|V\rangle_{q_{2}}+\gamma_{2}^{\prime}|H\rangle_{k_{2}}+\eta_{2}^{\prime}|V\rangle_{l_{2}}\right) \\
= & -\frac{\gamma_{1} \gamma_{2}}{4}(\alpha|V V\rangle+\beta|H H\rangle)_{p_{1} p_{2}}+\frac{\eta_{1} \eta_{2}}{4}(\alpha|H H\rangle-\beta|V V\rangle)_{q_{1} q_{2}}+\frac{\gamma_{1}^{\prime} \gamma_{2}^{\prime}}{4}(-\alpha|V V\rangle+\beta|H H\rangle)_{k_{1} k_{2}} \\
& +\frac{\eta_{1}^{\prime} \eta_{2}^{\prime}}{4}(-\alpha|H H\rangle+\beta|V\rangle)_{l_{1} l_{2}}+\frac{i \gamma_{1} \eta_{2}}{4}(\alpha|V H\rangle+i \beta|H V\rangle)_{p_{1} q_{2}}+\frac{i \gamma_{1} \gamma_{2}^{\prime}}{4}(i \alpha|V V\rangle+\beta|H H\rangle)_{p_{1} k_{2}} \\
& +\frac{i \gamma_{1} \eta_{2}^{\prime}}{4}(i \alpha|V H\rangle+\beta|H V\rangle)_{p_{1} l_{2}}+\frac{i \gamma_{2} \eta_{1}}{4}(\alpha|H V\rangle+i \beta|V H\rangle)_{q_{1} p_{2}}+\frac{i \eta_{1} \gamma_{2}^{\prime}}{4}(\alpha|H V\rangle+\beta|V H\rangle)_{q_{1} k_{2}} \\
& +\frac{i \eta_{1} \eta_{2}^{\prime}}{4}(\alpha|H H\rangle+\beta|V V\rangle)_{q_{1} l_{2}}+\frac{i \gamma_{1}^{\prime} \eta_{2}}{4}(\alpha|V H\rangle+\beta|H V\rangle)_{k_{1} q_{2}}+\frac{i \gamma_{1}^{\prime} \gamma_{2}}{4}(i \alpha|V V\rangle+\beta|H H\rangle)_{k_{1} p_{2}} \\
& +\frac{\eta_{2}^{\prime} \gamma_{1}^{\prime}}{4}(-\alpha|V H\rangle+\beta|H V\rangle)_{k_{1} l_{2}}+\frac{i \gamma_{2} \eta_{1}^{\prime}}{4}(i \alpha|H V\rangle+\beta|V H\rangle)_{l_{1} p_{2}}+\frac{i \eta_{1}^{\prime} \eta_{2}}{4}(\alpha|H H\rangle+\beta|V V\rangle)_{l_{1} q_{2}} \\
& +\frac{\eta_{1}^{\prime} \gamma_{2}^{\prime}}{4}(-\alpha|H V\rangle+\beta|V H\rangle)_{l_{1} k_{2}} .
\end{aligned}
$$

In reality, each photon includes four possible ports and a total of $4^{2}$ combinations may be obtained. From Equation (12), we know that the success probability for polarization modes is $\frac{1}{4}$. Taking the spatial mode into consideration, the overall probability for obtaining a pair of uncorrupted hyperentangled photons is $\frac{1}{16}$.

\section{DISCUSSION AND CONCLUSION}

Generalizing our error-rejecting transmission scheme is straightforward in the case of hyperentangled $n$-photon systems. Suppose that the initial state of hyperentangled $n$ photons is

$$
|\psi\rangle=\left(\alpha^{\prime}\left|a_{1} a_{2} \cdots a_{n}\right\rangle+\beta^{\prime}\left|b_{1} b_{2} \cdots b_{n}\right\rangle\right)\left(\alpha\left|H_{1} H_{2} \cdots H_{n}\right\rangle+\beta\left|V_{1} V_{2} \cdots V_{n}\right\rangle\right) .
$$

By applying Equation (9), the evolution of the spatial state is

$$
\begin{aligned}
&|\psi\rangle_{s}=\alpha^{\prime}\left|a_{1} a_{2} \cdots a_{n}\right\rangle+\beta^{\prime}\left|b_{1} b_{2} \cdots b_{n}\right\rangle \\
& \rightarrow \frac{\alpha^{\prime}}{2^{n}} \prod_{j=1}^{n}\left(i e^{i \theta_{A_{j}}}|a\rangle_{\delta t A^{j}}-e^{i \theta_{B_{j}}}|a\rangle_{\delta t B^{j}}\right)+\frac{\beta^{\prime}}{2^{n}} \prod_{j=1}^{n}\left(i e^{i \theta_{A_{j}}}|b\rangle_{\delta t A^{j}}+e^{i \theta_{B_{j}}}|b\rangle_{\delta t B^{j}}\right) \\
&= \sum_{m=0}^{n} \sum_{\forall a_{1}, \cdots, a_{m}} \frac{e^{i \Theta}}{2^{n}}\left(\alpha^{\prime}(-1)^{n-m} i^{m}|a\rangle_{A^{1}}|a\rangle_{A^{2}} \cdots|a\rangle_{A^{m}}|a\rangle_{B^{1}} \cdots|a\rangle_{B^{n-m}}\right. \\
&\left.\quad+\beta^{\prime} i^{m}|b\rangle_{A^{1}}|b\rangle_{A^{2}} \cdots|b\rangle_{A^{m}}|b\rangle_{b^{1}} \cdots|b\rangle_{b^{n-m}}\right)
\end{aligned}
$$

Here the symbol $\sum_{\forall a_{1}, \cdots, a_{m}}$ means the sum of all the occasions when $\mathrm{m}$ photons arrive at path A while n-m photons arrive at path B. For simplicity, we replace various phase shifts by a uniform term $e^{i \Theta}$. For each photon, a consistent one-to-one match between the states $|a\rangle$ and $|b\rangle$ may be noted. Thus, a total of $\sum_{m=0}^{n}\left(\begin{array}{c}n \\ m\end{array}\right)=2^{n}$ combinations is achieved, which offers a success probability of $\frac{1}{2^{n}}$. For the polarization mode, using Equation (11), we get

$$
\begin{aligned}
|\psi\rangle_{p}= & \alpha\left|H_{1} H_{2} \cdots H_{n}\right\rangle+\beta\left|V_{1} V_{2} \cdots V_{n}\right\rangle \\
\rightarrow & \frac{\alpha^{\prime}}{2^{n}} \prod_{j=1}^{n}\left(i \gamma_{j}|V\rangle_{p^{j}}+\eta_{j}|H\rangle_{q^{j}}+\gamma_{j}^{\prime} i|V\rangle_{k^{j}}+i \eta_{j}^{\prime}|H\rangle_{l^{j}}\right)+\frac{\beta^{\prime}}{2^{n}} \prod_{j=1}^{n}\left(i \gamma_{j}|H\rangle_{p^{j}}+i \eta_{j}|V\rangle_{q^{j}}+\gamma_{j}^{\prime}|H\rangle_{k^{j}}+\eta_{j}^{\prime}|V\rangle_{l^{j}}\right) \\
= & \sum_{a+b+c+d=n} \frac{\gamma \eta}{2^{n}}\left(\alpha i^{a+c+d}|V\rangle_{p^{1}} \cdots|V\rangle_{p^{a}}|H\rangle_{q^{1}} \cdots|H\rangle_{q^{b}}|V\rangle_{k^{1}} \cdots|V\rangle_{k^{c}}|H\rangle_{l^{1}} \cdots|H\rangle_{l^{d}}\right. \\
& \left.\quad+\beta i^{a+b}|H\rangle_{p^{1}} \cdots|H\rangle_{p^{a}}|V\rangle_{q^{1}} \cdots|V\rangle_{q^{b}}|H\rangle_{k^{1}} \cdots|H\rangle_{k^{c}}|V\rangle_{l^{1}} \cdots|V\rangle_{l^{d}}\right) .
\end{aligned}
$$


Notice that $\gamma$ and $\eta$ represent the collective noise on different channels, the total success probability for transmitting $\mathrm{n}$ photons in spatial-polarization hyperentangled mode is $\frac{1}{4^{n}}$.

Hyperentanglement presents good applicability in high-capacity quantum communication. The proposed self-errorrejecting photonic qubit transmission scheme can actively reject the collective noise in optical channels. Similar to the case where hyperentanglement purification [44-48] and hyperentanglement concentration [49 58] are more complicated than entanglement purification [59 67] and entanglement concentration [68 84] for photon systems in only one DOF, this self-error-rejecting scheme for hyperentangled photon systems is more complicated than the self-error-rejecting scheme for photon systems in only the polarization DOF [19]. However, it is an active method that allows hyperentangled photon systems to overcome the collective noise in channels. Combining hyperentanglement purification and hyperentanglement concentration [44 58], it may be very useful for high-capacity quantum communication as this scheme works with linear-optical elements and can, in principle, be implemented easily with current experimental techniques.

In our self-error-rejecting scheme for photon systems in two DOFs, we assume the efficiency of the linear-optical elements, including PBSs, BSs, and HWPs, to be perfect, i.e., no photon loss occurs in these linear-optical elements. In practical applications of this self-error-rejecting scheme, the elements do not work in ideal conditions, and photon loss is one of the main challenges for its application. Another challenge that must be addressed is the accurate time delay of all over relatively long period of times, which will affect the success probability of the interference between two wave packets in decoders. In principle, these two main challenges can be overcome with optical chips, which can integrate linear-optical elements to control the delay time accurately and depress photon loss.

In summary, we have proposed a self-error-rejecting qubit transmission scheme for an N-photon system in both the polarization and spatial states over noisy channels using linear-optical elements only. The polarization and spatial states are easily influenced by the collective noise. By converting these states into different time bins, the influence of noise could be transformed into a global phase, thereby enabling easy selection of uncorrupted states during the detection process. Moreover, our scheme promises a stable success probability without suffering from the influence of the coefficients of noisy channels. This scheme may be very useful in practical high-capacity quantum communication with photons in two or more DOFs.

\section{ACKNOWLEDGMENTS}

This work is supported by the National Natural Science Foundation of China under Grants No. 61675028 and No. 11674033, the Fundamental Research Funds for the Central Universities under Grant No. 2015KJJCA01, and the National High Technology Research and Development Program of China under Grant No. 2013AA122902.

[1] C. H. Bennett, G. Brassard, C. Crépeau, R. Jozsa, A. Peres, and W. K. Wootters, Phys Rev Lett. 70, 1895 (1993).

[2] Q. Ai, Sci Bull. 61, 110 (2016).

[3] C. H. Bennett and S. J. Wiesner, Phys Rev Lett. 69, 2881 (1992).

[4] X. S. Liu, G. L. Long, D. M. Tong, and F. Li, Phys Rev A. 65, 022304 (2002).

[5] C. H. Bennett and G. Brassard, Proceedings of IEEE International Conference on Computers, Systems and Signal Processing (Bangalore, 1984) P.175.

[6] A. K. Ekert, Phys Rev Lett. 67, 661 (1991).

[7] C. H. Bennett, G. Brassard, and N. D. Mermin, Phys Rev A. 68, 557 (1992).

[8] X. H. Li, F. G. Deng, and H. Y. Zhou, Phys Rev A. 78, 022321 (2008).

[9] M. Hillery, V. Bužek, and A. Berthiaume, Phys Rev A. 59, 1829 (1999).

[10] G. L. Long and X. S. Liu, Phys Rev A. 65, 032302 (2002).

[11] F. G. Deng, G. L. Long, and X. S. Liu, Phys Rev A. 68, 042317 (2003).

[12] F. G. Deng and G. L. Long, Phys Rev A. 69, 052319 (2004).

[13] C. Wang, F. G. Deng, Y. S. Li, X. S. Liu, and G. L. Long, Phys Rev A. 71, 044305 (2005).

[14] J. Y. Hu, B. Yu, M. Y. Jing, L. T. Xiao, S. T. Jia, G. Q. Qin, and G. L. Long, Light: Sci Appl. 5, e16144 (2016).

[15] W. Zhang, D. S. Ding, Y. B. Sheng, L. Zhou, B. S. Shi, and G. C. Guo, Phys Rev Lett. 118, 220501 (2017).

[16] X. H. Li, Acta Phys Sin. 64, 160307 (2015).

[17] D. Kalamidas, Phys Lett A. 343, 331 (2005).

[18] T. Yamomoto, J. Shimamura, S. K. Ozdemir, M. Koashi, and N. Imoto, Phys Rev Lett. 95, 040503 (2005).

[19] X. H. Li, F. G. Deng, and H. Y. Zhou, Appl Phys Lett. 91, 1444101 (2007).

[20] F. G. Deng, B. C. Ren, and X. H. Li, Sci Bullet. 62, 46 (2017).

[21] J. T. Barreiro, N. K. Langford, N. A. Peters, and P. G. Kwiat, Phys Rev Lett. 95, 260501 (2005).

[22] M. Barbieri, C. Cinelli, P. Mataloni, F. De Martini, Phys Rev A. 72, 052110 (2005). 
[23] R. Ceccarelli, G. Vallone, F. De Martini, P. Mataloni, and A. Cabello, Phys Rev Lett. 103, 160401 (2009).

[24] G. Vallone, R. Ceccarelli, F. De Martini, and P. Mataloni, Phys Rev A. 79, 030301(R) (2009).

[25] A. Rossi, G. Vallone, A. Chiuri, F. De Martini, and P. Mataloni, Phys Rev Lett. 102, 153902 (2009).

[26] D. Bhatti, J. von Zanthier, and G. S. Agarwal, Phys Rev A. 91, 062303 (2015).

[27] Y. B. Sheng, F. G. Deng, and G. L. Long, Phys Rev A. 82, 032318 (2010).

[28] B. C. Ren, H. R. Wei, M. Hua, T. Li, and F. G. Deng, Opt Express. 20, 24664 (2012).

[29] T. J. Wang, Y. Lu, and G. L. Long, Phys Rev A. 86, 042337 (2012).

[30] Q. Liu and M. Zhang, Phys Rev A. 91, 062321 (2015).

[31] X. H. Li and S. Ghose, Phys Rev A. 93, 022302 (2016).

[32] G. Y. Wang, Q. Ai, B. C. Ren, T. Li, and F. G. Deng, Opt Express. 24, 28444 (2016).

[33] B. C. Ren and F G. Deng, Sci Rep. 4, 4623 (2014).

[34] B. C. Ren, G. Y. Wang, and F. G. Deng, Phys Rev A. 91, 032328 (2015).

[35] T. Li and G. L. Long, Phys Rev A. 94, 022343 (2016).

[36] B. C. Ren and F. G. Deng, Opt Express. 25, 10863 (2017).

[37] J. T. Barreiro, T. C. Wei, and P. G. Kwiat, Nat Phys. 4, 282 (2008).

[38] Y. B. Sheng and F. G. Deng, Phys Rev A. 81, 032307 (2010).

[39] Y. B. Sheng and F. G. Deng, Phys Rev A. 82, 044305 (2010).

[40] X. H. Li, Phys Rev A. 82, 044304 (2010).

[41] F. G. Deng, Phys Rev A. 83, 062316 (2011).

[42] Y. B. Sheng and L. Zhou, Sci Rep. 5, 7815 (2015).

[43] T. J. Wang, S. Y. Song, and G. L. Long, Phys Rev A. 85, 062311 (2012).

[44] B. C. Ren and F. G. Deng, Laser Phys Lett. 10, 115201 (2013).

[45] B. C. Ren, F. F. Du, and F. G. Deng, Phys Rev A. 90, 052309 (2014).

[46] G. Y. Wang, Q. Liu, and F. G. Deng, Phys Rev A. 94, 032319 (2016).

[47] F. F. Du, T. Li, and G. L. Long, Ann Phys. 375, 105 (2016).

[48] T. J. Wang, L. L. Liu, R. Zhang, C. Cao, and C. Wang, Opt Express. 23, 9284 (2015).

[49] B. C. Ren, F. F. Du, and F. G. Deng, Phys Rev A. 88, 012302 (2013).

[50] B. C. Ren and G. L. Long, Opt Express. 22, 6547 (2014).

[51] X. H. Li and S. Ghose, Laser Phys Lett. 11, 125201 (2014).

[52] X. H. Li and S. Ghose, Opt Express. 23, 3550 (2015).

[53] X. H. Li and S. Ghose, Phys Rev A. 91, 062302 (2015).

[54] B. C. Ren and G. L. Long, Sci Rep. 5, 16444 (2015).

[55] C. Cao, T. J. Wang, S. C. Mi, R. Zhang, and C. Wang, Ann Phys. 369, 128 (2016).

[56] L. L. Fan, Y. Xia, and J. Song, Quantum Inf Process. 13, 1967 (2014).

[57] H. J. Liu, Y. Xia, and J. Song, Quantum Inf Process. 15, 2033 (2016).

[58] F. F. Du, F. G. Deng, and G. L. Long, Sci Rep. 6, 35922 (2016).

[59] C. H. Bennett, G. Brassard, S. Popescu, B. Schumacher, J. A. Smolin, and W. K. Wootters, Phys Rev Lett. 76, 722 (1996).

[60] J. W. Pan, C. Simon, Č. Brukner, and A. Zeilinger, Nature. 410, 1067 (2001).

[61] C. Simon and J. W. Pan, Phys Rev Lett. 89, 257901 (2002).

[62] Y. B. Sheng, F. G. Deng, and H. Y. Zhou, Phys Rev A. 77, 042308 (2008).

[63] C. Wang, Y. Zhang, and G. S. Jin, Phys Rev A. 84, 032307 (2011).

[64] Y. B. Sheng, L. Zhou, G. L. Long, Phys Rev A. 88, 022302 (2013).

[65] T. Li, G. J. Yang, and F. G. Deng, Opt Express. 22, 23897 (2014).

[66] L. Zhou and Y. B. Sheng, Laser Phys Lett. 12, 045203 (2015).

[67] L. Zhou and Y. B. Sheng, Sci Rep. 6, 28813 (2016).

[68] C. H. Bennett, H. J. Bernstein, S. Popescu, and B. Schumacher, Phys Rev A. 53, 2046 (1996).

[69] Z. Zhao, J. W. Pan, and M. S. Zhan, Phys Rev A. 64, 014301 (2001).

[70] T. Yamamoto, M. Koashi, and N. Imoto, Phys Rev A. 64, 012304 (2001).

[71] Y. B. Sheng, F. G. Deng, and H. Y. Zhou, Phys Rev A. 77, 062325 (2008).

[72] Y. B. Sheng, L. Zhou, S. M. Zhao, and B. Y. Zheng, Phys Rev A. 85, 012307 (2012).

[73] F. G. Deng, Phys Rev A. 85, 022311 (2012).

[74] Y. B. Sheng, L. Zhou, and S. M. Zhao, Phys Rev A. 85, 042302 (2012).

[75] F. F. Du and F. G. Deng, Sci China-Phys Mech Astron. 58, 040303 (2015).

[76] C. Cao, C. Wang, L. Y. He, and R. Zhang, Opt Express. 21, 4093 (2013).

[77] C. Wang, W. W. Shen, S. C. Mi, Y. Zhang, and T. J. Wang, Sci Bull. 60, 2016 (2015).

[78] C. Cao, X. Chen, Y. W. Duan, L. Fan, R. Zhang, T. J. Wang, C. Wang, Sci China-Phys Mech Astron. 59, 100315 (2016).

[79] Y. B. Sheng, J. Pan, R. Guo, L. Zhou, and L. Wang, Sci China-Phys Mech Astron. 58, 060301 (2015).

[80] F. F. Du and G. L. Long, Quantum Inf Process. 16, 26 (2017).

[81] H. J. Liu, L. L. Fan, Y. Xia, and J. Song, Quantum Inf Process. 14, 2909 (2015).

[82] A. Banerjee, C. Shukla, A. Pathak, Quantum Inf Process. 14, 4523 (2015).

[83] C. Shukla, A. Banerjee, A. Pathak, Quantum Inf Process. 14, 2077 (2015).

[84] J. Pan, L. Zhou, S. P. Gu, X. F. Wang, Y. B. Sheng, and Q. Wang, Quantum Inf Process. 15, 1669 (2016). 